\title{
A Fractional-Order Primal-Dual Denoising Algorithm
}

\author{
Dan Tian \\ School of Information Engineering \\ Shenyang University \\ Shenyang, China \\ www.sltd2008@163.com \\ Dapeng Li \\ School of Information Engineering \\ Shenyang University \\ Shenyang, China \\ 295677734@qq.com
}

\author{
Yingxin Zhang \\ School of Information Engineering \\ Shenyang University \\ Shenyang, China \\ 735497145@qq.com
}

\begin{abstract}
Objective: By combining fractional calculus and duality theory, a novel fractional-order primal-dual model which is equivalent with the fractional ROF model is proposed. We theoretically analyze its structural similarity with the saddle-point optimization model. So the algorithms for solving the saddle-point problem can be used for solving the model. Methods: The primal-dual algorithm based on resolvent for solving the saddle-point problem is used for solving the proposed model. The adaptive variable step size iterative optimization strategy is used, which can improve the optimizing efficiency, and remedy the step size limitation of the traditional numerical algorithms. In order to guarantee the convergence of the algorithm, the range of the parameter is given. Results: The experiment results show that the proposed fractional-order primal-dual model is effective in avoiding the staircase effect and preserving texture and detail information, and the adoptive numerical algorithm has faster convergence speed. Conclusion: This paper proposes a fractional-order primal-dual denoising model, which can be solved by a primal-dual algorithm based on resolvent. The experiment results show that the proposed model can improve the image visual effect effectively, and the adoptive numerical algorithm has faster convergence speed.
\end{abstract}

Keywords- image denoising; fractional-order; primaldual; saddle-point problem; variation method

\section{INTRODUCTION}

Image denoising is a significant preliminary step in many machine vision tasks. The goal is to estimate an original image $u$ from an observed image $g$, modeled as

$$
\mathrm{g}=\mathrm{u}+\mathrm{n}
$$

where $\mathrm{n}$ is a vector of zero-mean Gaussian white noise with variance $\sigma^{2}$. A number of methods have been taken to estimate $\mathrm{u}$, including wavelet transform [1], partial differential equation [2], Fourier transform [3], and total variation [4], etc. where the total variation method is quite efficient for removing noise while preserving edges. One of the most well known total variation models was introduced by Rudin, Osher and Fatemi (ROF), which mathematically formulated the denoising problem as the following minimization [5]:

$$
\min _{u \in X}\|\nabla u\|_{1}+\frac{\lambda}{2}\|u-g\|_{2}^{2}
$$

where $X$ is the finite dimensional vector space, $\|\bullet\|_{v}$ is the $v$ norm, $\nabla$ is the gradient operator, $\lambda$ is the regularization parameter. However, the ROF model favors a piecewise constant solution which often leads to the staircase effect, and small details are often filtered out in the process of denoising. To solve this problem, the fractional-order derivative has been introduced to the total variation denoising models to replace the traditional firstorder regularization. Since the fractional-order derivative can consider more neighboring pixels information, which helps describe more image details.

In this paper, a primal-dual formulation of the fractional-order ROF model is given based on duality principle, which is found having the remarkable similar structure with the saddle-point problem. So it can be solved by a flexible primal-dual algorithm [6], which has been used for solving the saddle-point problem successfully. In [6], this primal-dual algorithm has been shown having rapid convergence compared with gradient descend algorithm [7], fixed-point iteration algorithm [8], shrinkage thresholding algorithm [9], etc.

\section{FRACTIONAL-ORDER DENOISING MODEL}

\section{A. Fractional-Order ROF Model}

Fractional-order derivative has been applied in various scientific fields. But its definition is not unified until now. In this paper, the GL definition[10] is used. Assume the size of image $u$ is $M \times N$, then the discrete form of $\nabla^{\alpha} u$ can be represented as

$$
\left(\nabla^{\alpha} u\right)_{i, j}=\left(\left(\Delta_{1}^{\alpha} u\right)_{i, j},\left(\Delta_{2}^{\alpha} u\right)_{i, j}\right) \quad 1 \leq i \leq M, 1 \leq j \leq N
$$

with 


$$
\left\{\begin{array}{l}
\left(\Delta_{1}^{\alpha} u\right)_{i, j}=\sum_{k=0}^{K-1}(-1)^{k} C_{k}^{\alpha} u_{i-k, j} \\
\left(\Delta_{2}^{\alpha} u\right)_{i, j}=\sum_{k=0}^{K-1}(-1)^{k} C_{k}^{\alpha} u_{i, j-k}
\end{array}\right.
$$

where $K \geq 3$ is an integer constant, $C_{k}^{\alpha}=\frac{\Gamma(\alpha+1)}{\Gamma(k+1) \Gamma(\alpha-k+1)}, \Gamma$ is the gamma function.

By generalizing the variation order from integer to fraction, the fractional-order ROF model can be obtained as

$$
\min _{u \in X}\left\|\nabla^{\alpha} u\right\|_{1}+\frac{\lambda}{2}\|u-g\|_{2}^{2}
$$

where

$$
\begin{gathered}
\left\|\nabla^{\alpha} u\right\|_{1}=\sum_{i, j}\left|\left(\nabla^{\alpha} u\right)_{i, j}\right| \\
\text { with } \\
\left|\left(\nabla^{\alpha} u\right)_{i, j}\right|=\sqrt{\left(\left(\Delta_{1}^{\alpha} u\right)_{i, j}\right)^{2}+\left(\left(\Delta_{2}^{\alpha} u\right)_{i, j}\right)^{2}}
\end{gathered}
$$

From the variation definition, an important property can be observed. The integer derivative just involves finite terms; However, the fractional derivative involves infinite number of terms. The fractional operator can consider more neighboring pixels information, preserving more image details.

\section{B. Fractional-Order Primal-Dual Model}

A fractional-order primal-dual model, i.e. the primaldual formulation of the fractional-order ROF model will be presented.

For $\quad p \in Y \quad, \quad$ let $p_{i, j}=\left(p_{i, j}^{1}, p_{i, j}^{2}\right) \quad, \quad$ and $\left|p_{i, j}\right|=\sqrt{\left(p_{i, j}^{1}\right)^{2}+\left(p_{i, j}^{2}\right)^{2}} \leq 1$, then

$$
\left\|\nabla^{\alpha} u\right\|_{1}=\sup _{p \in Y}<\nabla^{\alpha} u, p>
$$

Let $F(u)=\left\|\nabla^{\alpha} u\right\|_{1}$, then its conjugate function $F^{*}(p)$ satisfies the following property

$$
F_{P}^{*}(p)= \begin{cases}0 & p \in P \\ +\infty & p \notin P\end{cases}
$$

where $P=\left\{p \in Y:\|p\|_{\infty}=\max _{i, j}\left|p_{i, j}\right| \leq 1\right\}$ is the conjugate set.

The primal-dual formulation of the fractional-order ROF model can be given by

$$
\min _{u \in X} \max _{p \in Y}<\nabla^{\alpha} u, p>+\frac{\lambda}{2}\|u-g\|_{2}^{2}-F_{P}^{*}(p)
$$

\section{Saddle-Point Problem}

Using the Fenchel duality, the generic saddle-point problem can be formulated as

$$
\min _{x \in X} \max _{y \in Y}<A x, y>+G(x)-F^{*}(y)
$$

where $X$ and $Y$ are the finite dimensional real vector space, $<\cdot,>$ denotes the standard Euclidean inner product, $A$ is a random linear operator, $G$ and $F$ are lowersemicontinuous functions, $F^{*}$ is the conjugate of function $F$.

This saddle-point problem is the primal-dual formulation of the following primal problem

$$
\min _{x \in X} F(A x)+G(x)
$$

and the corresponding dual problem

$$
\max _{y \in Y}-\left(G^{*}\left(-A^{*} y\right)+F^{*}(y)\right)
$$

It is not hard to find that this saddle-point optimization model has the remarkable similar structure with the proposed denoising model (9), if $A=\nabla^{\alpha}$, $G(u)=\frac{\lambda}{2}\|u-g\|_{2}^{2}, F^{*}(p)=F_{P}^{*}(p)$.

\section{NUMERICAL COMPUTATION}

To solve the saddle-point problem (10), a primal-dual algorithm [6] based on resolvent has been proposed, and has been tested having rapid convergence compared with gradient descend algorithm, fixed-point iteration algorithm, shrinkage thresholding algorithm, etc. Fixing the primal variable $x$, taking the derivative of the dual variable $y$, the resolvent operator of variable $y$ can be obtained

$$
y=\left(I+\partial F^{*}\right)^{-1}(y+A x)
$$

Similarly, fixing the dual variable $y$, taking the derivative of the primal variable $x$, the resolvent operator of variable $x$ can be obtained

$$
x=(I+\partial G)^{-1}(x-A y)
$$

where $\partial F^{*}$ and $\partial G$ are the subgradients of the functions $F^{*}$ and $G$.Define the parameter $L$, which satisfies the following property

$$
L=\|A\|=\max \{\|A x\|: x \in X,\|x\| \leq 1\}
$$

then when $G$ or $F^{*}$ is uniformly convex, the primal-dual algorithm [6] can be summarized as follows:

I Initialization: Choose $\tau_{0}, \sigma_{0}>0$ with $\tau_{0} \sigma_{0} L^{2} \leq 1$, $\left(x^{0}, y^{0}\right) \in X \times Y$, and ${ }^{-0}=x^{0}$.

I Iterations $(n \geq 0)$ : Update as follows

$$
\left\{\begin{array}{l}
y^{n+1}=\left(I+\sigma_{n} \partial F^{*}\right)^{-1}\left(y^{n}+\sigma_{n} A x^{-n}\right) \\
x^{n+1}=\left(I+\tau_{n} \partial G\right)^{-1}\left(x^{n}-\tau_{n} A^{*} y^{n+1}\right) \\
\theta_{n}=1 / \sqrt{1+2 \gamma \tau_{n}}, \tau_{n+1}=\theta_{n} \tau_{n}, \sigma_{n+1}=\sigma_{n} / \theta_{n} \\
x^{n+1}=x^{n+1}+\theta_{n}\left(x^{n+1}-x^{n}\right)
\end{array}\right.
$$

I Stopping test: stop if

$$
\begin{gathered}
\varsigma(x, y)=\max _{y^{\prime} \in B_{2}}<y^{\prime}, A x>-F^{*}\left(y^{\prime}\right)+G(x)- \\
\min _{x^{\prime} \in B_{1}}<y, A x^{\prime}>-F^{*}(y)+G\left(x^{\prime}\right)
\end{gathered}
$$

where $\varsigma(x, y)$ is the difference between the dual problem and the primal problem, it vanishes only if $(u, p)$ is the saddle-point[9].

Considering the similar structure between the propsed fractional-order primal-dual denoising model and the saddle-point problem, and the algorithm precondition is met, i.e. $A=\nabla^{\alpha}$ is a linear operator, and the fidelity term $G(u)=\frac{\lambda}{2}\|u-g\|_{2}^{2}$ is a convex function, the denoising optimization procedure can be realized by the former primal-dual algorithm. 
In the numerical computation, it remains to detail the resolvent operators $\left(I+\partial F^{*}\right)^{-1}$ and $(I+\partial G)^{-1}$. Since $F^{*}(p)=F_{P}^{*}(p)$ and $G(u)=\frac{\lambda}{2}\|u-g\|_{2}^{2}$, one has

$$
\begin{gathered}
p=\left(I+\sigma \partial F^{*}\right)^{-1}(\tilde{p}) \Leftrightarrow p_{i, j}=\frac{\tilde{p_{i, j}}}{\max \left(1, \mid \tilde{p_{i, j} \mid}\right)} \\
u=(I+\tau \partial G)^{-1}(\tilde{u}) \Leftrightarrow u_{i, j}=\frac{\tilde{u}_{i, j}+\tau \lambda g_{i, j}}{1+\tau \lambda}
\end{gathered}
$$

where $\tilde{p}=p+\sigma A \bar{u}, \tilde{u}=u-\tau A^{*} p$.

Next, we will consider the convergence problem. [6] has proven the algorithm can convergent to the saddlepoint. Here, we only need to confirm the convergence condition, i.e. the value range of the parameter $L$. For every dual variable $p=\left(p^{1}, p^{2}\right) \in Y$, the discrete fractional-order divergence can be defined as

$$
d i v^{\alpha} p=\left(d i v^{\alpha} p\right)_{i, j} \quad i=1,2, \ldots, M, \quad j=1,2, \ldots, N
$$

with

$$
\left(d i v^{\alpha} p\right)_{i, j}=(-1)^{\alpha} \sum_{k=0}^{K-1}(-1)^{k} C_{k}^{\alpha} p_{i+k, j}^{1}+(-1)^{\alpha} \sum_{k=0}^{K-1}(-1)^{k} C_{k}^{\alpha} p_{i, j+k}^{2}
$$

since

$$
\begin{aligned}
&\left\|\overline{(-1)^{\alpha}} d i v^{\alpha} p\right\|^{2}=\sum_{i, j}\left(w_{0} p_{i, j}^{1}+w_{1} p_{i+1, j}^{1}+\mathrm{L}+w_{K-1} p_{i+K-1, j}^{1}+\right. \\
&\left.w_{0} p_{i, j}^{2}+w_{1} p_{i, j+1}^{2}+\mathrm{L}+w_{K-1} p_{i, j+K-1}^{2}\right)^{2} \leq \\
& 2 K \times \sum_{i, j}\left(w_{0} p_{i, j}^{1}\right)^{2}+\left(w_{0} p_{i, j}^{2}\right)^{2}+\left(w_{1} p_{i+1, j}^{1}\right)^{2}+ \\
&\left(w_{1} p_{i, j+1}^{2}\right)^{2}+\mathrm{L}+\left(w_{K-1} p_{i+K-1, j}^{1}\right)^{2}+ \\
&\left(w_{K-1} p_{i, j+K-1}^{2}\right)^{2} \leq 2 K \times\left(w_{0}^{2}+w_{1}^{2}+\mathrm{L}+w_{K-1}^{2}\right)\|p\|^{2} \leq \\
& 2 K \times\left(w_{0}^{2}+w_{1}^{2}+\mathrm{L}+w_{K-1}^{2}\right)
\end{aligned}
$$

Hence

$$
L=\left|\nabla^{\alpha}\right|=\left\|\overline{(-1)^{\alpha}} d i v^{\alpha}\right\| \leq \sqrt{2 K \times\left(w_{0}^{2}+w_{1}^{2}+\mathrm{L}+w_{K-1}^{2}\right)}
$$

where $w_{i}=(-1)^{i} C_{i}^{\alpha}, K$ is the number of terms in the fractional-order derivative definition.

\section{NUMERICAL RESUlts}

In this section, we provide numerical results to show the behavior of the proposed fractional-order primal-dual model with respect to its denoising capabilities. In the following, all test images are treated as column vector with $\mathrm{M} \times \mathrm{N}$ elements via row-wise scanning way. Using row-wise scanning method, we can map a pixel value at position $(i, j)$ of an image into the $[(i-1) \times N+j]$ element of the vector.

Considering the frequency characteristic of fractional differential, we generalize the traditional first-order ROF model to the fractional-order one. Fig. 1 shows the amplitude-frequency response of differential, when $\alpha \in[1.0,3.0]$.

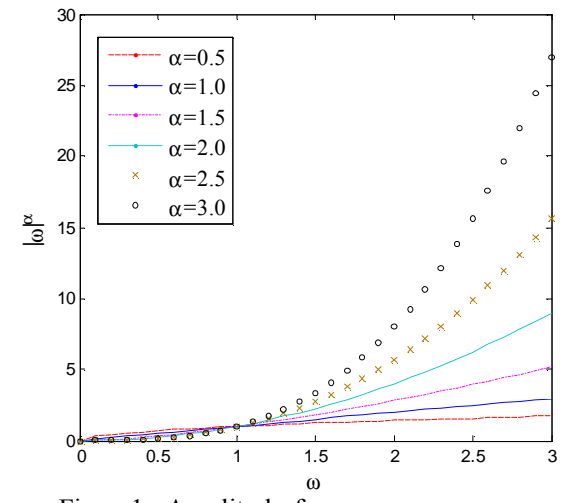

Figure1. Amplitude-frequency response

It is observed that in the medium frequency and the high frequency sections, corresponding to images' texture and edge area, the enhancement degree of images with fractional differential operator is much better than the ones produced by the first-order operator. This implies that the fractional ROF model should be used to enhance the medium frequency texture and the high frequency edge. Considering the performance of both denoising and details-perserving, $\alpha=1.0: 2.0$ is the best choice.

To show the role of fractional differential in the proposed fractional-order primal-dual model, we fix the parameter $\lambda$, and select a cardiac ultrasound image with size $156 \times 156$ as test image. Let $\mathrm{n}=200, \lambda=10, \alpha$ be selected from 0 to 3 . Experiment results show that the larger $\alpha$ is, the better image details are preserved, but meanwhile the more noise is residual too. Considering the performance of both denoising and details-perserving, $\alpha=1.0: 2.0$ is the best choice. Fig. 2 lists the denoised images under different fractional order.

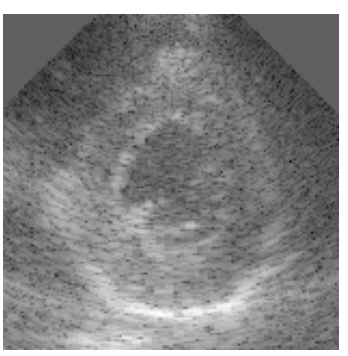

(a)

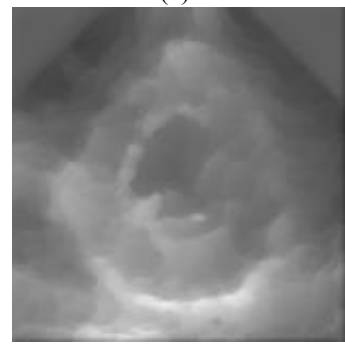

(c)

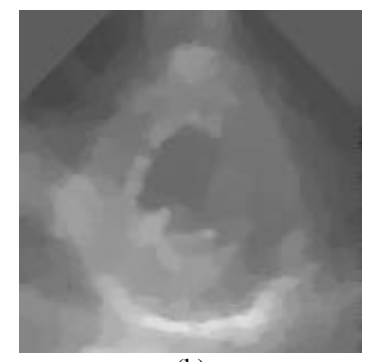

(b)

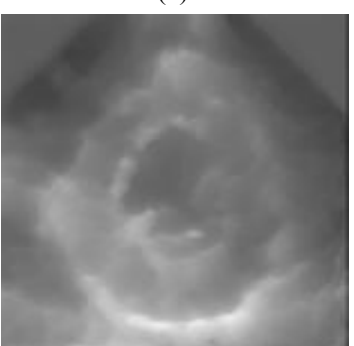

(d) 


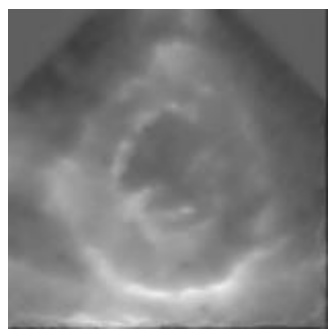

(e)

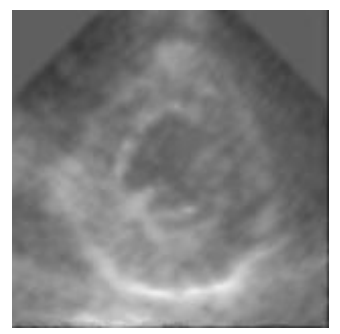

(f)

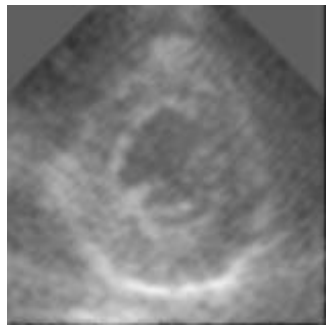

(g)

Figure 2. Comparison of denoising results under different fractional order on a cardiac ultrasound image. (a) Original image, (b) $\alpha=1.0$, (c) $\alpha=1.2$, (d) $\alpha=1.4$, (e) $\alpha=1.6$, (f) $\alpha=1.8$, (g) $\alpha=2.0$.

It is clearly seen that the fractional order differential can eliminate the staircase effect and preserve some small details compared with the first order differential. But along with the increasing of $\alpha$, the more noise is residual too.

\section{CONCLUSIONS}

We develop a fractional-order primal-dual model and the accompanying numerical algorithm for image denoising problem. The numerical results show that the proposed model yields better results with respect to the protection of image details.

\section{ACKNOWLEDGEMENTS}

The work is supported by the China National Nature Science Foundation (Grant No.61174145) and the China Liaoning Province Education Department Science and Technology Research Project (Grant No. L2013448).

\section{REFERENCES}

[1] X. H. Han and X. M. Chang, "An Intelligent Noise Reduction Method for Chaotic Signals Based on Genetic Algorithms and Lifting Wavelet Transforms," Information Sciences, vol. 218, Jan. 2013, pp.103-118.

[2] P. Liu, F. Huang, and G. Li, "Remote-Sensing Image Denoising Using Partial Differential Equations and Auxiliary Images as Priors," IEEE Geoscience and Remote Sensing Letters, vol. 9, Mar. 2012, pp.358-362.

[3] M. D. Robinson, C. A. Toth, J. Y. Lo, and S. Farsiu, "Efficient Fourier-Wavelet Super-Resolution," IEEE Transactions on Image Processing, vol.19, Oct. 2010, pp.2669-2681.

[4] F. Arandiga, P. Mulet, and V. Renau, "A Fast Primal-Dual Method for Generalized Total Variation Denoising," Applied Mathematics, vol. 6, Mar. 2012, pp.401-409.

[5] L. I. Rudin, S. Osher, and F. E, "Nonlinear Total Variation Based Noise Removal Algorithms," Physica D, vol. 60, 1/2/3/4. 1992, pp. 259-268.

[6] A. Chambolle and T. Pock, "A First-Order Primal-Dual Algorithm for Convex Problems with Applications to Imaging," Journal of Mathematical Imaging and Vision, vol. 40, Jan. 2011, pp. 120-145.

[7] A. Beck, M. Teboulle, "Fast Gradient-Based Algorithms for Constrained Total Variation Image Denoising and Deblurring problems," IEEE Trans.on Image Processing, vol.18, Nov. 2013, pp. 2419-2434.

[8] Y. W. Wen and R. H. Chan, "Parameter Selection for TotalVariation-Based Image Restoration Using Discrepancy Principle," IEEE Transactions on Image Processing, vol. 21, Apr. 2012, pp. 1770-1781.

[9] A. Beck and M. Teboulle, "A Fast Iterative Shrinkage Thresholding Algorithm for Linear Inverse Problems," SIAM Journal on Imaging Sciences, vol.2, Jan. 2009, pp. 183-202.

[10] I. Odlubny, Fractional differential equations. New York: Academic Press, 1999. 